\begin{tabular}{|l|c|c|c|}
\hline & & $170-176$ & Hannover 1998 \\
\hline
\end{tabular}

\title{
Die phylogenetische Stellung der Neandertaler im Licht erster DNA-Analysen
}

\author{
Ralf W. Schmitz \& Heike Krainitzki*) \\ Neandertals, continuity or replacement, DNA preservation, Neandertal DNA sequence, sequence \\ analyses, separate species, replacement
}

\begin{abstract}
Kurzfassung: Das Neandertal liegt 13 Kilometer östlich von Düsseldorf, Deutschland. Im August $1856 \mathrm{ka}-$ men beim Abbau der Kleinen Feldhofer Grotte Teile eines menschlichen Skelettes zutage. In den folgenden Jahren entbrannte vor dem Hintergrund der Evolutionstheorie eine heftige Kontroverse um Alter und Einstufung der Entdeckung

Heute wird der Fund allgemein den Späten Neandertalern der letzten Kaltzeit zugerechnet. Über die phylogenetische Stellung und das Schicksal dieser Menschen bestehen sehr unterschiedliche Auffassungen.

Seit 1991 wird das Neandertaler-Typusexemplar im Rahmen eines interdisziplinären Projektes des Rheinischen Landesmuseums Bonn neu untersucht. Recherchen in Bezug auf Faktoren wie z. B. die Lagerungsbedingungen des Skelettes in der Grotte, weiterhin der sehr gute Erhaltungszustand der geborgenen Skelettreste ließen die Suche nach DNA vertretbar erscheinen. Die besonders gute Erhaltung des rechten Humerus einhergehend mit dem guten Zustand der schützenden Lackschicht auf der Knochenoberfläche führten zur Entscheidung, diesen Knochen zu beproben.

Aus dieser Probe konnte eine mitochondriale DNA-Sequenz des Neandertalers isoliert werden.

Sequenzvergleiche mit mitochondrialen DNA-Sequenzen moderner Menschen zeigen, daß die NeandertalerSequenz aus der Variationsbreite heutiger Menschen herausfällt.

Die Neandertaler-Sequenz stützt ein Szenario, in dem die modernen Menschen als getrennte Spezies in Afrika entstanden sind und später die Neandertaler ohne oder nahezu ohne Vermischung ersetzt haben.
\end{abstract}

\section{[The phylogenetic status of the Neandertals by means of first DNA analyses]}

Abstract: The Neander Valley lies 13 kilometers east of Düsseldorf in Germany. In August 1856 parts of a human skeleton come to light during the quarrying of the 'Kleine Feldhofer Grotte'.

In the years following a fierce controversy flared up against the background of the theory of evolution as to the age and the classification of the discovery.

Today the find is attributed to the Late Neandertals of the last glacial period. There exists very different views

*) Anschrift der Verfasser: Dr. R. W. Schmitz, Landschaftsverband Rheinland, Rheinisches Amt für Bodendenkmalpflege, Endenicher Str. 133, 53115 Bonn, H. KRAINITZKI, Höhere Berufsfachschule für präparationstechnische Assistenten, Markstraße 185, 44799 Bochum as to the phylogenetic status and the fate of these beings.

Since 1991, the Neandertal-type specimen has been the subject of an interdisciplinary project of the Rheinisches Landesmuseum Bonn.

Research relating to factors, for example the bedding conditions of the skeleton in the grotto and furthermore the very high degree of preservation of the recovered skeletal remains, allowed the search for DNA appear justifiable.

The particularly good condition of the right humerus hand in hand with the good condition of the protective varnish on the bone surface, led to the decision to take a sample from this bone.

From this sample an Neandertal mitochondrial DNA sequence was derived.

Sequence comparisons with modern human mitochondrial DNA sequences show that the Neandertal sequence falls outside the variation of modern humans.

The Neandertal sequence supports a scenario in which modern humans arose in Africa as a distinct species and replaced Neandertals with little or no interbreeding.

\section{Einleitung}

Das Neandertal liegt 13 Kilometer östlich von Düsseldorf, Nordwestdeutschland. Die Düssel hatte hier, verursacht durch die tektonische Heraushebung des mitteldevonischen Massenkalkes, eine enge Schlucht eingeschnitten. In den bis zu 60 Meter hohen Klippen befanden sich vor der Zerstörung durch Kalksteinbrüche die Eingänge zu mehreren Höhlen und Grotten.

Im August 1856 kamen beim Abbau der auf dem südlichen Düsselufer gelegenen Kleinen Feldhofer Grotte Teile eines menschlichen Skelettes zutage. Geborgen wurden 15 postcraniale Knochen und die Schädelkalotte eines männlichen Erwachsenen (Abb. 1). Der Fund wurde durch den Elberfelder Lehrer und Naturforscher JOHANN CARL FuHLROTT als Menschenrest erkannt und einer umfassenden wissenschaftlichen Bearbeitung zugeführt. In den folgenden Jahren entbrannte vor dem Hintergrund der Evolutionstheorie (DARWIN 1859) eine heftige Kontroverse um Alter und Einstufung der Entdeckung. 


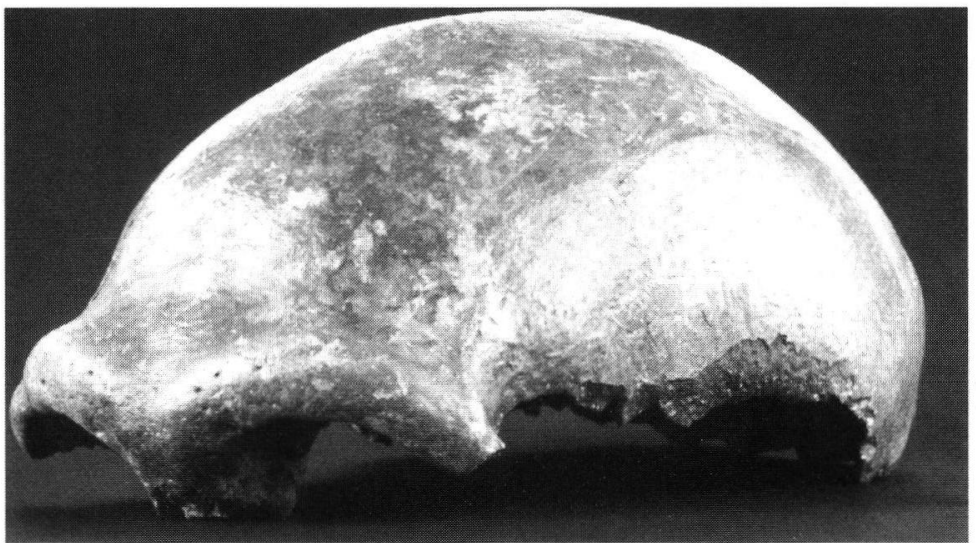

Abb. 1: Schädelkalotte des Neandertaler-Typusexemplars.

Fig. 1: Skull Calotte of the Neandertal-Type Specimen.

(Photo: H. Lilienthal, Rheinisches Landesmuseum Bonn).

tionen von Homo erectus entwickelt hat. Für Europa wird postuliert, daß die Neandertaler Vorfahren der heutigen Europäer sind (z. B. Wolpoff et al. 1994; Wolpoff \& CASPARI 1997).

Hingegen sehen die Vertreter des 'Out of Africa II'-Modells die Entwicklung zum anatomisch modernen Menschen ausschließlich in Afrika und nehmen an, daß die Neandertaler durch spätestens vor 40000 Jahren nach Europa eingewanderte anatomisch moderne Menschen ersetzt wurden. Weiterhin wird angenom-

Während z. B. Fuhlrotт (1859), der Bonner Anatom Hermann Schaffhausen $(1857,1888)$, Thomas Henry Huxley (1863: 119-159) und Charles Lyell (1863: 75-92) von einem sehr hohen Alter der Entdeckung ausgingen oder ein solches zumindest in Erwägung zogen, gab es zahlreiche Deutungen, die sich gegen diese Einstufung richteten. Am bekanntesten ist die von Rudolf Virchow vertretene These, die morphologischen Abweichungen vom anatomisch modernen Menschen seien pathologisch bedingt (1872).

1863/64 beschäftigte sich der Ire WILLIAM KING mit dem Fund und setzte ihn mit der Benennung Homo neanderthalensis als eigene Art von allen späteren Menschen ab (KING 1864).

Es ist sehr wahrscheinlich, daß im Neandertal mit der Kleinen Feldhofer Grotte eine Bestattung durch den Kalkabbau zerstört wurde und dabei der größte Teil des Skelettes verloren ging; bedingt durch die Art der Bergung existieren weder eine Dokumentation noch Begleitfunde.

Seit dem Tode FuntrotT's 1877 befinden sich die Skelettreste im Rheinischen Landesmuseum Bonn. Das damalige Provinzialmuseum erwarb sie auf Vermittlung SchaffHausen's, um so einen Verkauf ins Ausland zu verhindern, den FuHLRotT stets abgelehnt hatte.

Heute wird der Fund allgemein den von Europa bis Westasien verbreiteten, Späten Neandertalern der letzten Kaltzeit zugerechnet.

Über die phylogenetische Stellung und das Schicksal dieser Menschen bestehen sehr unterschiedliche Auffassungen:

Die Vertreter des Multiregionalen Modells gehen davon aus, daß sich an verschiedenen Orten der Welt der anatomisch moderne Mensch (Homo sapiens) jeweils aus archaischen Vorgängerpopula- men, daß die Neandertaler keinen oder nur einen minimalen genetischen Beitrag zur Entstehung der heutigen Europäer geleistet haben (z. B. Stringer 1992; Stringer \& Gamble 1993: 193; Howell 1994; BrÄUER 1992; BrÄUER \& BROEG 1998). Eine afrikanische Genese des anatomisch modernen Menschen und eine spätere, wesentliche Vermischung mit den Neandertalern wird ebenfalls diskutiert (Sмгтн 1992, 1994).

\section{Chancen für die Erhaltung alter DNA in Neandertaler-Fossilien}

Seit 1991 wird das Typusexemplar im Rahmen eines durch einen der Verfasser (R. W. SchmiTz) initiierten und geleiteten interdisziplinären Projektes des Rheinischen Landesmuseums Bonn umfassend neu untersucht (SchmiTz 1996: 191-205). Seit Mitte 1992 war das Zoologische Institut der Universität München, zunächst im Rahmen eines Gedankenaustausches über die Chancen einer Gen-Analyse, am Projekt beteiligt.

In den neunziger Jahren waren bereits mehrere Versuche verschiedener Laboratorien, Genmaterial aus Neandertaler-Fossilien zu gewinnen, ohne Erfolg geblieben. Da weiterhin der heute übliche Aminosäuretest zur vorbereitenden Überprüfung potentieller DNA-Erhaltung (POINAR et al. 1996) noch nicht zur Verfügung stand, waren mehrjährige Recherchen der Verfasser in Bezug auf die Chance einer Überlieferung von DNA im Skelettmaterial aus der Kleinen Feldhofer Grotte im Neandertal unabdingbar. Bei Neufunden ist es möglich, derartige Recherchen auf primäre Faktoren, wie etwa die Lagerungsbedingungen, zu beschränken; bei Altfunden tritt hingegen ein gravierendes Problem in Erscheinung: Die nicht sel- 
ten undokumentierte Behandlung in den Jahrzehnten nach der Bergung.

So waren z. B. die Techniken und Materialien in den Bereichen Konservierung und Abgußtechnik von 1856 bis heute einem steten Wandel unterworfen. Gerade bei Altfunden wie dem Neandertaler murs davon ausgegangen werden, daß in den Jahren nach seiner Entdeckung immer wieder verschiedene Techniken zum Einsatz kamen und damit einhergehend auch eine Reihe diverser Substanzen Verwendung fanden. Die Restauratoren, Präparatoren und Konservatoren haben zwar nach den jeweiligen Möglichkeiten ihrer Zeit stets eine optimale Behandlung angestrebt, doch sind die verwendeten Stoffe nicht immer verträglich mit den teils lange Zeit nach ihrer Anwendung entwickelten wissenschaftlichen Untersuchungsmethoden

Daher wurden alle gebräuchlichen Techniken und Materialien der letzten 140 Jahre in Betracht gezogen und nach Spuren für deren Anwendung am Neandertalerfund gesucht. Einige Hinweise fanden sich auf den Überresten des Neandertalers selbst, andere in der zeitgenössischen Literatur (FLHLROTT 1865: 68: LYELL 1864: 44, 57: SCHAFFHAUSEN 1888: 5, 9, 23, 41: SCHWALbe 1901: 1, 3, 38). Es wurde eine Liste mit Auffälligkeiten und Hinweisen erstellt und mit den zur Anwendung kommenden Untersuchungsmethoden abgestimmt. Insgesamt konnte eine Reihe von Faktoren herausgearbeitet werden, welche die Suche nach DNA des Neandertalers vertrethar erscheinen ließsen: diese Faktoren dürften zugleich den Schlüssel für die Erhaltung alter DNA im Knochenmaterial des Typusexemplars darstellen:

1. Die Einbettung des Individuums erfolgte etwa $60 \mathrm{~cm}$ tief im Sediment einer sehr kleinen Grotte (Länge ca. 4,70 m, Breite ca. 3,10 m, Höhe von der Sedimentoberkante ca. 1,20 m). Der Felsboden der Grotte war beckenförmig vertieft (Funlrot 1859: 135), so daß das etwa 1,60-1,90 m mächtige Sedimentpaket besonders geschützt gelagert war.

Hinzu kommt, daß der „Eingang” 1856 lediglich etwa $60 \times 20 \mathrm{~cm}$ maß (FuHLrott 1868: 63). Diese für einen Erwachsenen nicht passierbare Öffnung legt einen Versturz unbestimmte Zeit nach der Bestattung nahe. Insgesamt ist von einer weitgehenden Abschottung des Höhlensedimentes von Witterungseinflüissen auszugehen.

2. Die Grotte war nordexponiert (FuHLROTT 1859: 135); aus einer von BONGARI 1835 verfaßten Beschreibung der Schlucht geht hervor, daß die entsprechende Felswand stets im Schatten lag. Ohnehin stellt sich selbst bei kleinen Höhlen die Raumtemperatur auf die jeweilige Jahresdurchschnittstemperatur der entsprechenden Region ein (Moore \& Sullivas 1980: 28). Diese liegt im Bergischen Land heute bei etwa $10^{\circ} \mathrm{C}$; während des größten Teils der Lagerungsdauer war der Grottenraum der Kleinen Feldhofer Grotte den klimatischen Verhältnissen der letzten Kaltzeit unterworfen, so daß von einer noch wesentlich geringeren Höhlenraumtemperatur auszugehen ist.

3. Das Fundsediment wird als homogene, den Knochen fest anhaftende, dichte Lehmmasse beschrieben (FUHLROTT 1859: 136, 137), die den Einfluß von Luftsauerstoff auf die Skelettreste minimiert haben wird.

4. Durch die Lage der Grotte im Massenkalk ist von einem basischen Einbettungsmilieu der Skelettreste auszugehen.

5. Durch die bis in die Gegenwart andauernde tektonische Heraushebung des Rheinischen Schiefergebirges und damit des Neandertaler Massenkalkes stiegen auch die Höhlen und Grotten über das Bachniveau der Düssel auf, so daß sie selbst bei Hochwasser nicht mehr überflutet werden konnten. So gibt FunLrotT (1859: 134) z. B. für die Kleine Feldhofer Grotte eine Höhe von rund 20 Metern über dem Talboden an.

6. Bereits Schaffhalsfon (1857, 1888) diagnostizierte für den Neandertaler eine in der Jugend erlittene Verletzung des linken Armes mit anschließender, dauerhafter Beugebeschränkung desselben. Die hieraus resultierende, unterschiedliche Einsatzfähigkeit der beiden Arme führte zu einer Schwächung des linken Humerus; hingegen ist die Knochensubstanz des rechten Humerus deutlich kräftiger ausgebildet. Die Diagnose wurde u.a. durch entsprechende Röntgenbefunde (M. Schultz, Zentrum Anatomie, Abt. Morphologie, Univ. Göttingen), bestätigt.

7. Zwar sind für die Schädelkalotte Manipulationen durch den mittelpaläolithischen Menschen in Form von Schnittspuren belegt (CzARNETZKI 1977; SCHMITZ \& PIEPER 1992; SCHMITZ 1996: 194-202), die größeren Langknochen weisen jedoch keine Fragmentierung durch Zerschlagen auf.

Die relativ geringe Einbettungstiefe des Fundes führte dazu, daß ein Zerbrechen dieser Knochen durch Sedimentdruck ebenfalls aus- 
blieb; schließlich überstand der robuste Humerus dext. auch die Entfernung der Grottenfüllung durch die Steinbrucharbeiter ohne relevante Beschädigungen. Hieraus folgt, daß die Angriffsfläche für DNA-schädigende Einflüsse sowohl während der Lagerung im Sediment als auch nach der Bergung wesentlich geringer war als dies für fragmentierte Knochenfunde anzunehmen ist.

8. Der wie beschrieben sehr gut erhaltene Humerus dext. erhielt bereits relativ kurze Zeit nach der Bergung, nach erfolgter erster Abformung, einen Lacküberzug, bei dem es sich um Schellack handelt, einem Ausscheidungsprodukt der Schildlaus. In den 30er Jahren dieses Jahrhunderts wurde er nochmals abgeformt und erhielt einen weiteren Überzug mit dem für die damalige Zeit üblichen Celluloselack. Diese, wenn auch nach heutigen konservatorischen Maßstäben nicht zu befürwortende Behandlung durch diverse Lacke sprach dafür, daß das Knochenmaterial des Humerus dext. vor Verunreinigungen durch das häufige Hantieren in den vergangenen 140 Jahren weitgehend geschützt war.

9. Für eine Durchtränkung des Knochenmaterials mit heißem Leim fanden sich keine Anhaltspunkte. Relevant ist in diesem Zusammenhang auch eine Aussage SchaffHausev's (1888: 27): „Für das Alter der Neanderthaler Knochen kann auch der Umstand angeführt werden, dass ein Stück Rippe und ein Stück vom Becken in verdünnter Salzsäure 11\% organ. Materie und eine nicht bestimmte Menge von Leim zurückliessen, was nach Kestner's schöner Entdeckung eine Eigenthümlichkeit alter, zumal fossiler Knochen ist." SchaffHadsen hat diesen Test genutzt, um das fossile Alter des Neandertalerfundes zu untermauern; eine die Reproduzierbarkeit dieses Versuchs verunmöglichende Konservierung des Skelettes mit Leim hätte kaum die Billigung des wissenschaftlichen Erstbearbeiters erfahren.

Mit steigender Akzeptanz gegenüber der Evolutionstheorie bestand jedoch nicht mehr die Notwendigkeit für eine solche Beweisführung und es wurden Empfehlungen zur Konservierung von Knochenmaterial ausgesprochen (FrAAs 1910: 8; Hauser 1917: 133; RathgeN 1924; 1926). Daher sollte bei einer Beprobung, insbesondere von Altfunden, nicht allein ein Aminosäuretest des Fossils vorgeschaltet sein, da ein durch den Test festgestellter guter molekularer Erhaltungszustand des Knochens durch rezent über Konservierungsmittel eingebrachte Aminosäuren vorgetäuscht sein kann.

Zusammenfassend ergab sich die Annahme, daß potentielle DNA des Neandertalers insbesondere im Humerus dext. (Inventarnummer 322, 09) des namengebenden Neandertalers durch die aufgeführten Faktoren vor schädlichen Einflüssen (z. B. größere Mengen Wasser, hohe Temperaturen, saures Milieu) sehr gut geschützt war und eine wesentliche Verunreinigung des vielhantierten Altfundes mit rezenter DNA kaum zu befürchten sei.

Bei der daraufhin im Juni 1996 durch die Präparatorin des Projektes (H. KrainITZKI) aus diesem Knochen steril entnommenen Probe handelte es sich um eine 1, $4 \mathrm{~cm}$ dicke Halbscheibe (Abb. 2) mit einem Gewicht von 3, 5 Gramm.

Die nachfolgenden, mehrere Monate in Anspruch nehmenden Experimente wurden durch $M$. KrINGs und S. PÄÄBO am Zoologischen Institut der Universität München ausgeführt. Diese Arbeiten

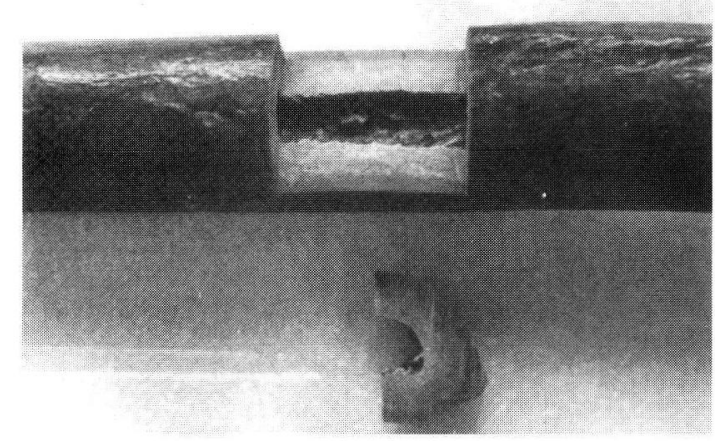

Abb. 2: Probe, entnommen aus dem rechten $\mathrm{Hu}-$ merus des Neandertaler-Typusexemplars.

Fig. 2: Sample Removed from the Right Humerus of the Neandertal-Type Specimen.

(Photo: H. Krainitzki).

zielten nicht ab auf DNA aus den Zellkernen, sondern aus den Mitochondrien, also den ,Zellkraftwerken". Da auf einen Satz Kern-DNA einige tausend der wesentlich kürzeren mitochondrialen DNA-Stränge entfallen, ist die Erfolgschance bei einer Suche nach entsprechender DNA entsprechend größer.

Die Münchener Analysen erbrachten in mehreren, unabhängigen Durchgängen aus der Probe des Typusexemplars die erste DNA-Sequenz eines Neandertalers.

Das Ergebnis wurde anschließend durch M. STONEKING und A. STONE am Department of Anthropology, Pennsylvania State University, USA, wiederholt und bestätigt. 


\section{Sequenz und Interpretation}

Die nachgewiesene Sequenz (Länge 379 Basen) stellt bei einem geschätzten Alter von 40000 bis 80000 Jahren - die Datierung des Fossils ist in Vorbereitung - das bisher älteste untersuchte menschliche Genmaterial dar.

Der Vergleich von 994 heute lebenden Menschen untereinander ergab, daß diese sich, unabhängig von ihrer geographischen Herkunft, im analysierten Sequenz-Abschnitt auf durchschnittlich acht Positionen unterscheiden. Im Vergleich mit dem untersuchten Neandertaler sind es hingegen im Mittel 27 Positionen (Abb. 3). Damit fällt dieser Neandertaler aus der Variationsbreite der mitochondrialen DNA der heute auf der Erde lebenden Menschen heraus.

Basierend auf dem genetischen Abstand zwischen heutigem Mensch und Schimpanse sowie der Annahme, daß der letzte gemeinsame Vorfahre der beiden vor etwa 4-5 Millionen Jahren lebte, wurden entsprechende Berechnungen für den Neandertaler und jetzt lebende Menschen durch-

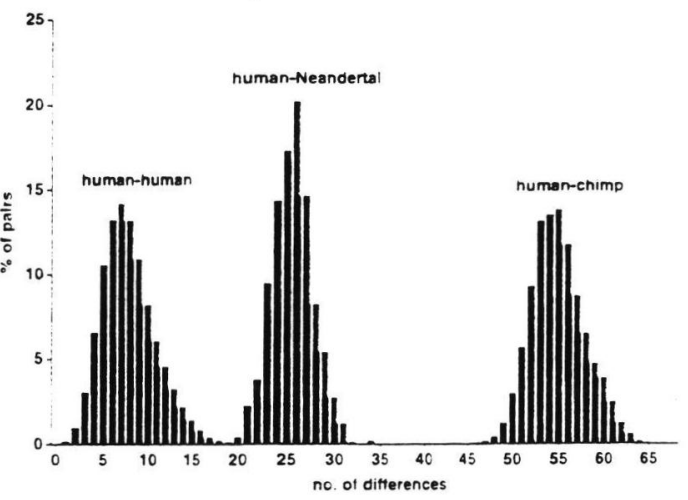

Abb. 3: Verteilungen von paarweisen SequenzUnterschieden zwischen rezenten Menschen, dem Neandertaler, und Schimpansen.

Fig. 3: Distributions of Pairwise Sequence Differences among recent Humans, the Neandertal, and Chimpanzees. (Krings et al. 1997).

geführt. Es zeigte sich, daß für deren letzten gemeinsamen Vorfahren ein Alter von 690000 bis 550000 Jahren wahrscheinlich ist (KRINGs et al. 1997).

Es ist davon auszugehen, daß dieser Vorfahre der entwickelte Homo erectus war.

In Afrika folgen auf diesen zunächst der frühe und späte archaische Homo sapiens, ab etwa 150000 der anatomisch moderne Homo sapiens (BRÄUER et al. 1997).

Europa brachte hingegen eine eigenständige, vom entwickelten Homo erectus ausgehende Li- nie hervor, welche schließlich zu den Späten Neandertalern der Weichsel-Kaltzeit führte.

Der anatomisch moderne Mensch und der Späte Neandertaler stellen somit die am weitesten voneinander entfernten Endpunkte zweier getrennter Entwicklungslinien dar.

Auch konnte gezeigt werden, daß der untersuchte Neandertaler von den heute auf verschiedenen Kontinenten lebenden Menschen gleich weit entfernt ist, also keinesfalls mit modernen Europäern eine engere Verwandtschaft aufweist als mit irgendeiner anderen modernen Population.

Dieses Ergebnis steht in klarem Widerspruch zum Multiregionalen Modell, während es das 'Out of Africa II'-Modell stützt (Krings et al. 1997).

Sollten sowohl die Analysen der restlichen 1996 entnommenen Probensubstanz auf weitere Sequenzabschnitte, als auch Arbeiten an anderen Neandertalern und Fossilien des anatomisch modernen Menschen verschiedener Provenienz sowie unterschiedlicher Zeitstellung die ersten Ergebnisse bestätigen, ist davon auszugehen, daß die Späten Neandertaler ohne Beitrag mitochondrialer DNA zum aktuellen menschlichen Genpool ausstarben, also nicht zu den Vorfahren der jetzt lebenden Menschen gehören.

Die Annahme einer in Europa entstandenen Biospezies Homo neanderthalensis mit entsprechenden Reproduktionsbarrieren gegen den anatomisch modernen Homo sapiens würde hierdurch nachdrücklich unterstrichen.

Für die Ablösung der Neandertaler durch die anatomisch modernen Menschen in Europa ist folgendes Szenario denkbar:

Vor mindestens 40000 Jahren erreichte Homo sapiens, ausgehend vom Vorderen Orient - hier waren die Neandertaler bereits abgelöst worden zunächst Südosteuropa und drang im weiteren Verlauf dieser ersten, bis etwa 35000 dauernden Phase bis nach Frankreich vor.

In dieser Zeit gab es ein Jahrtausende währendes Nebeneinander von Neandertalern und modernen Menschen bei noch langsamem Schwinden des Neandertaler-Genpools.

Es ist von Interaktion zwischen den unterschiedlichen Individuen und Gruppen sowie wechselseitiger kultureller Beeinflussung auszugehen.

Ein Beleg für diese Annahme dürften z. B. die Funde von Arcy-sur-Cure (Grotte du Renne) sein. Hier konnten neben Steinartefakten des Châtelperronien Schmuckgegenstände geborgen werden, wie sie ansonsten von Fundplätzen des 
Homo sapiens bekannt sind. Daß es sich bei den Menschen des Châtelperronien um Neandertaler handelte, ist durch die Skelettreste von Arcysur-Cure und Saint-Césaire in Frankreich gut dokumentiert (Hublin et al. 1996; ApSimon 1980; Mercier et al. 1991).

Daß die progressiven Elemente in der Artefakttechnologie des Châtelperronien ebenfalls eine Akkulturationserscheinung darstellen, ist möglich, aber zu ihrer Erklärung nicht notwendig. Progressive Elemente wie eine systematische Klingenproduktion und die Fertigung kleiner, rückengestumpfter Steinartefakte, die sich als Einsätze in Kompositgeräte eignen, finden sich bereits in mitteleuropäischen Inventaren, die aufgrund ihrer frühen Zeitstellung (später Eem-Interglazialkomplex) nicht mit dem anatomisch modernen Menschen in Verbindung gebracht werden können. Hierzu zählen u. a. Tönchesberg 2B in der Osteifel (stage $5 \mathrm{e} / 5 \mathrm{~d}$; CONARD 1992) und Mönchengladbach-Rheindahlen B1 (stage 5c?; Schmitz \& Thissen, im Druck). Diese und weitere Inventare sprechen für eine hohe, autochthone Variabilität des europäischen Mittelpaläolithikums.

In der Phase des Nebeneinander von Neandertaler und Homo sapiens könnte es zu Vermischungen gekommen sein. Waren die hieraus resultierenden Nachkommen ihrerseits jedoch infertil, so ließe sich diese Vermischung im heutigen Genpool nicht nachweisen. Der gesicherte Nachweis solcher im Vergleich zur Gesamtbevölkerung wohl nicht sehr zahlreichen Hybriden dürfte am immer noch geringen europäischen Fossilbestand aus dieser Zeit kaum zu erbringen sein.

Ab etwa 35000 kam es durch die weitere Ausbreitung von Homo sapiens offensichtlich $\mathrm{zu}$ einer extremen Abdrängung der Neandertaler in die Randgebiete Europas; die jüngsten Hinweise finden sich im Süden der Iberischen Halbinsel. In der Höhle von Zafarraya konnte u.a. ein auf etwa 32000 Jahre datierter Unterkiefer eines Neandertalers entdeckt werden; Moustérien-Geräte aus jüngeren Schichten des selben Fundortes sind rund 27000 Jahre alt (Huburn et al. 1995). Sind diese Daten korrekt, so dürften die Funde von Zafarraya zu den letzten Spuren der Neandertaler gehören.

Mit dieser weiteren Verdrängung wird eine zunehmende Ausdünnung des Genpools einhergegangen sein, so daß schließlich die Spezies Homo neanderthalensis nachkommenlos verloschen ist.

\section{Danksagung}

Wir danken Prof. Dr. F. G. ZeHnder und Prof. Dr. H.- E. JoAchim, Direktion des Rheinischen Landesmuseums Bonn, für vielfältige Unterstützung sowie die Genehmigung der Probenentnahme; unser Dank gilt ebenfalls dem früheren Direktor des Museums, Dr. H. LüDTKE, für seine Unterstützung. Mein (R.W.S.) ganz besonderer Dank gilt meinem leider inzwischen verstorbenen Doktorvater Prof. Dr. W. TAuTE, ehemals Institut für Ur- und Frühgeschichte der Universität zu Köln.

\section{Schriftenverzeichnis}

ApSimon, A. M. (1980): The last Neanderthal in France?Nature 287: 271-272, 2 Abb.; London (Macmillan).

BONGARD, J. H. (1835): Wanderung zur Neandershöhle. Eine topographische Skizze der Gegend von Erkrath an der Düssel.- 67 S., 3 Abb.; Düsseldorf (Arnz \& Co.).

Bräuer, G. (1992): Africa's place in the evolution of Homo sapiens.- In: Bräuer, G. \& SMitH, F. H. (eds.): Continuity or Replacement. Controversies in Homo sapiens Evolution: 83-98, 3 Abb.; Rotterdam (Balkema).

Bräuer, G., Yokoyama, Y., Falguères, C. \& Mbua, E. (1997): Modern human origins backdated.- Nature 386: 337-338, 1 Abb., 1 Tab.; London (Macmillan).

Bräuer, G. \& Broeg, H. (1998): On the degree of Neandertal-Modern continuity in the earliest Upper Palaeolithic crania from the Czech Republic: Evidence from non-metrical features. - In Омото, K. \& Tobias, P. V. (eds): The Origins and Past of Modern Humans - Towards Reconciliation: 106-125, 9 Abb., 2 Tab.; Singapur.

CONARD, N. J. 1992: Tönchesberg and its position in the paleolithic prehistory of northern Europe.- Monographien des RGZM 20, XI + 176 S., 21 Tab., 13 Taf., 1 Beil; Bonn (Habelt).

Czarnetzki, A. (1977): Artefizielle Veränderungen an den Skelettresten aus dem Neandertal?- In: ScHrÖTER, P. (Hrsg.): 75 Jahre Anthropologische Staatssammlung München: 215-219, 4 Abb.; München (Anthropologische Staatssammlung).

Darwin, C. (1859): On the Origin of Species by Means of Natural Selection, or the Preservation of Favoured Races in the Struggle for Life.- X + 502 S., 1 Beil.; London (Murray).

FraAs, E. (1910): Der Petrefaktensammler. Ein Leitfaden zum Sammeln und Bestimmen der Versteinerungen Deutschlands.- VI + 249 S., 139 Abb., 72 Taf.; Stuttgart (Lutz).

FuhlrotT, J. C. (1859): Menschliche Ueberreste aus einer Felsengrotte des Düsselthals. Ein Beitrag zur Frage über die Existenz fossiler Menschen.- Verh. naturhist. Ver. preuss. Rheinl. Westf. 16: 131-153, 1 Taf.; Bonn (Henry \& Cohen).

- (1865): Der fossile Mensch aus dem Neanderthal und sein Verhältniß zum Alter des Menschengeschlechts.- 78 S., 2 Abb.; Duisburg (Falk \& Volmer).

- (1868): Vortrag über die Kalksteinschichten in der unmittelbaren Umgebung der kleineren Feldhofer 
Grotte im Neanderthal, in welcher (Sommer 1856) fossile Reste eines menschlichen Skelets, der sog. Homo Neanderthalensis, aufgefunden wurden.Verh. naturhist. Ver. preuss. Rheinl. Westf. 25, Corr. Bl.: 62-70; Bonn (Henry \& Cohen).

Halser, O. (1917): Der Mensch vor 100000 Jahren. 142 S., 96 Abb., 3 Kt.; Leipzig (Brockhaus).

Heרke, W. \& Rothe, H. (1994): Paläoanthropologie.XIII + 699 S., 304 Abb.; Berlin / Heidelberg (Springer).

How fll, F. C. (1994): A Chronostratigraphic and Taxonomic Framework of the Origins of Modern humans.- In: Nitecki, M. H. \& Nitecki D. V. (eds.): Origins of Anatomically Modern Humans: 253-319, 6 Tab.; New York (Plenum).

Hublin, J.- J., Barroso Ruiz, C., Medica lara, P., FontlgNE, M. \& Reyss, J.- L. (1995): The Mousterian site of Zafarraya (Andalucia, Spain): dating and implications on the palaeolithic peopling processes of Western Europe.- C. R. Acad. Sc. Paris 321 IIa: 931-937; Paris.

Hublix, J.- J., Spoor, F., Braly, M., Zosweveld, F. \& Condeni, S. (1996): A late Neanderthal associated with Upper Palaeolithic artefacts.- Nature 381: 224226, 3 Abb., 1 Tab.: London (Macmillan).

Huxlfy, T. H. (1863): Evidence as to Man's Place in Nature.- VI + 159 S., 32 Abb.; London (Williams and Norgate)

King, W. (1864): The Reputed Fossil Man of the Neanderthal.- The Quarterly Journal of Science 1: 88-97, 2 Taf.; London.

Krivgis, M., Stone, A., Schyitz. R. W., Kramitzki, H., STOveking, M. \& PÄ̈во, S. (1997): Neandertal DNA Sequences and the Origin of Modern Humans.- Cell 90/1: 19-30, 7 Abb., 1 Tab.; Cambridge, Mass. (Cell Press).

Lyell, C. (1863): The Geological Evidences of the Antiquity of Man with Remarks on Theories of the Origin of Species by Variation.- XII + 520 S., 58 Abb., 2 Taf.; London (Murray).

- (1864): Das Alter des Menschengeschlechts auf der Erde und der Ursprung der Arten durch Abänderung, nebst einer Beschreibung der Eiszeit in Europa und Amerika.- Mit Anmerkungen des Übersetzers L. BüChNer.- IX + 472 S., 57 Abb., 2 Taf.; Leipzig (Thomas).

Mercier, N., Valladas, H., Joron, J.- L., Reyss, J.- L., LÉvêque, F. \& VA.NDERMEersch, B. (1991): Thermoluminescence dating of the late Neanderthal remains from Saint-Césaire.- Nature 351: 737-739; London (Macmillan).

Moore, G. W. \& Sullivay, G. N. (1980): Speleology. The study of caves.- (2. Aufl.), 150 S.; Teaneck, N. Y. (Zephyrus Press).

PoI\ar, H. N., Höss, M., Bada, J. L. \& PÄ̈BO, S. (1996): Amino acid racemization and the preservation of ancient DNA.- Science 272: 864-866, 1 Abb., 2 Tab.; Washington (AAAS).

Rathgex, F. (1924): Die Konservierung von Altertumsfunden mit Berücksichtigung ethnographischer und kunstgewerblicher Sammlungsgegenstände. 2 Teil: Metalle und Metallegierungen, organische Stoffe.- Handbücher der staatlichen Museen zu Ber- lin, VII + 174 S., 65 Abb.; Berlin / Leipzig (de Gruyter).

- (1926): Die Konservierung von Altertumsfunden mit Berücksichtigung ethnographischer und kunstgewerblicher Sammlungsgegenstände. 1 Teil: Stein und steinartige Stoffe.- Handbücher der staatlichen Museen zu Berlin, X + 170 S., 98 Abb.; Berlin / Leipzig (de Gruyter ).

SchaAfFhausfo, H. (1857): 'Vortrag vom 2. Juni zu den Menschenknochen aus dem Neandertal'.- Verh. naturhist. Ver. preuss. Rheinl. Westf. 14, Corr. Bl.: 5052; Bonn (Henry \& Cohen).

- (1888): Der Neanderthaler Fund.- 49 S., 10 Abb., 3 Taf:; Bonn (Marcus).

Schmiтz, R. W. (1996): Das Alt- und Mittelpaläolithikum des Neandertals und benachbarter Gebiete.- XII + 253 S., 79 Abb., 94 Taf.; Dissertation, Köln.

- \& Pieper, P. (1992): Schnittspuren und Kratzer. Anthropogene Veränderungen am Skelett des Urmenschenfundes aus dem Neandertal - Vorläufige Befundaufnahme.- Das Rheinische Landesmuseum Bonn 2/92: 17-19, 6 Abb.; Köln (Rheinland-Verlag).

— \& Thissex, J. (1998): Vorbericht über die Grabungen 1995-1997 in der mittelpaläolithischen B1-Fundschicht der Ziegeleigrube Dreesen in Rheindahlen.Archäologisches Korrespondenzblatt, im Druck; Mainz (Verl. d. RGZM).

Schwalbe, G. (1901): Der Neanderthalschädel.- Bonner Jahrbücher 106: 1-72, 10 Abb., 1 Taf.; Bonn (Marcus \& Weber).

SмrTu, F. H. (1992): The role of continuity in modern human origins.- In: Bräter, G. \& SMiTH. F. H. (eds.): Continuity or Replacement. Controversies in Homo sapiens Evolution: 145-156, 3 Abb.; 1 Tab.; Rotterdam (Balkema).

- (1994): Samples, Species, and Speculations in the Study of Modern Human Origins.- In: NiTECki, M. H. \& NiteCkI, D. V. (eds.): Origins of Anatomically Modern Humans: 227-249, 1 Abb., 2 Tab.; New York (Plenum).

Stringer, C. B. (1992): Replacement, continuity and the origin of Homo sapiens.- In: BrÄuer, G. \& SмITH, F. H. (eds.): Continuity or Replacement. Controversies in Homo sapiens Evolution: 9-24, 4 Abb.; 1 Tab.; Rotterdam (Balkema).

- \& Gamble, C. (1993): In Search of the Neanderthals. Solving the Puzzle of Human Origins.- 247 S., 83 Abb., 100 Fot.; New York (Thames and Hudson).

Virchow, R. (1872): Untersuchung des Neanderthal Schädels.- Z. Ethnol. 4: 157-165; Berlin (Wiegandt und Hempel).

Wolpoff, M. H., Thorne, A. G., Smith, F. H., Frayer, D. W. \& Pope, G. G. (1994): Multiregional Evolution: A World-Wide Source for Modern Human Populations. In: Nitecki, M. H. \& NitFCki, D. V. (eds.): Origins of Anatomically Modern Humans: 175-199; New York (Plenum).

- \& CASPARI, R. (1997): Race and Human Evolution.462 S., 58 Abb.; New York (Simon \& Schuster).

Manuskript eingegangen am 13. Februar 1998 